\title{
Percepção de puérperas sobre o uso dos métodos não farmacológicos para alívio da
}

\section{dor no trabalho de parto}

\author{
Perception of puerperal women on the use of non-pharmacological methods for pain relief in \\ childbirth work
}

Percepción de las puérperas sobre el uso de métodos no farmacológicos para el alivio del dolor durante el trabajo de parto

Recebido: 05/04/2021 | Revisado: 12/04/2021 | Aceito: 23/04/2021 | Publicado: 08/05/2021

\author{
Aysla Kalliny dos Reis \\ ORCID: https://orcid.org/0000-0002-1649-2156 \\ Universidade Federal de Alagoas, Brasil \\ E-mail: ayslla_kaliny@hotmail.com \\ Jovânia Marques de Oliveira e Silva \\ ORCID: https://orcid.org/0000-0001-7452-2651 \\ Universidade Federal de Alagoas, Brasil \\ E-mail: jovaniasilva@gmail.com \\ Marcela Natal Lucena \\ ORCID: https://orcid.org/0000-0001-6150-4200 \\ Prefeitura Municipal do Recife, Brasil \\ E-mail: marcelanatallucena@gmail.com
}

\begin{abstract}
Resumo
Objetivo: Descrever a percepção de puérperas acerca do uso dos métodos não farmacológicos para alívio da dor no trabalho de parto e parto. Metodologia: Trata-se de um estudo descritivo com abordagem qualitativa, realizado em uma Maternidade pública do município de Recife - PE. Foram entrevistadas 10 puérperas que fizeram uso dos métodos não farmacológicos de alívio da dor durante o trabalho de parto e parto. Os dados foram produzidos por meio de entrevistas semiestruturada e analisados conforme a técnica de análise do conteúdo proposta por Bardin. Resultados: A partir da análise das falas das participantes emergiram quatro categorias temáticas: conhecimento sobre os métodos não farmacológicos, sensação de bem estar e alívio da dor, diminuição do tempo de trabalho de parto e valorização do acompanhante. Conclusão: $\mathrm{O}$ uso dos métodos não farmacológicos de alívio da dor tiveram efeitos positivos contribuindo na experiência do trabalho de parto e parto. Destaca-se a importância de estimular a autonomia da mulher desde o acompanhamento pré-natal na atenção básica, bem como dispor de uma equipe acolhedora e capacitada que forneça as informações e orientações necessárias contribuindo para a segurança da mulher e do seu acompanhante.
\end{abstract}

Palavras-chave: Métodos não farmacológicos; Trabalho de parto; Alívio da dor; Enfermagem obstétrica.

\begin{abstract}
Objective: To describe the perception of puerperal women about the use of non-pharmacological methods for pain relief during labor and delivery. Methodology: This is a descriptive study with a qualitative approach, carried out in a Public Maternity Hospital in the city of Recife - PE. 10 puerperal women who used non-pharmacological methods of pain relief during labor and delivery were interviewed. The data were produced through semi-structured interviews and analyzed according to the content analysis technique proposed by Bardin. Results: From the analysis of the speeches of the participants, four thematic categories emerged: knowledge about non-pharmacological methods, a sense of well-being and pain relief, reduction in labor time and appreciation of the companion. Conclusion: The use of non-pharmacological methods of pain relief had positive effects contributing to the experience of labor and delivery. The importance of encouraging women's autonomy from prenatal care in primary care is highlighted, as well as having a welcoming and trained team, which provides the necessary information and guidance, contributing to the safety of the woman and her companion.
\end{abstract}

Keywords: Non-pharmacological methods; Labor; Pain relief; Obstetric nursing.

\section{Resumen}

Objetivo: Describir la percepción de las puérperas sobre el uso de métodos no farmacológicos para el alivio del dolor durante el trabajo de parto y el parto. Metodología: Se trata de un estudio descriptivo con abordaje cualitativo, realizado en una Maternidad Pública de la ciudad de Recife - PE. Se entrevistó a 10 puérperas que utilizaron métodos no farmacológicos de alivio del dolor durante el trabajo de parto y el parto. Los datos fueron producidos a través de 
entrevistas semiestructuradas y analizados según la técnica de análisis de contenido propuesta por Bardin. Resultados: Del análisis de los discursos de los participantes surgieron cuatro categorías temáticas: conocimiento sobre métodos no farmacológicos, sensación de bienestar y alivio del dolor, disminución del tiempo de trabajo y aprecio del acompañante. Conclusión: El uso de métodos no farmacológicos de alivio del dolor tuvo efectos positivos que contribuyeron a la experiencia del trabajo de parto y el parto. Se destaca la importancia de fomentar la autonomía de la mujer desde la atención prenatal en la atención primaria, así como contar con un equipo acogedor y capacitado, que brinde la información y orientación necesaria, contribuyendo a la seguridad de la mujer y su acompañante.

Palabras clave: Métodos no farmacológicos; Trabajo de parto; Alivio del dolor; Enfermería obstétrica.

\section{Introdução}

O parto é historicamente um evento natural, de caráter pessoal e privado compartilhado com outras mulheres e seus familiares. Durante muito tempo, as parteiras ou comadres, consideradas pessoas de confiança das gestantes ou de experiência conhecida na comunidade era quem desempenhavam a atividade de partejar (Brasil, 2001).

A partir do século XX, o parto passou a ser realizado em ambiente hospitalar, submetendo a mulher ao modelo biomédico, onde o profissional entende o parto como processo patológico e faz uso de fármacos e outros procedimentos de forma abusiva. A assistência à mulher durante o parto tornou-se assim, objeto de grande medicalização, o cenário de nascimento transformou-se rapidamente, tornando-se desconhecido para as mulheres e mais conveniente e asséptico para os profissionais de saúde. Neste contexto, a parturiente encontra-se cada vez mais afastada da sua condição de protagonista do parto (Brasil, 2001).

A dor do parto faz parte da própria natureza humana e, ao contrário de outras experiências dolorosas agudas e crônicas, não está associada à patologia, mas sim, com a experiência de trazer uma nova vida. No entanto, algumas mulheres consideram que é a pior dor sentida e, muitas vezes, superior ao que esperavam (Brasil, 2001). O não esclarecimento a respeito do trabalho de parto, medo, tensão, solidão e estar em ambiente diferente e com pessoas estranhas, são considerados fatores que aumentam a percepção dolorosa no parto (Moraes, 2010).

No que tange a temática do alívio da dor à parturiente, o uso dos métodos não farmacológicos são propostos como uma opção para substituição de anestésicos e analgésicos durante o trabalho de parto e parto. E têm a finalidade de tornar o parto o mais natural possível, diminuindo as intervenções desnecessárias (Sescato et al.,2008). Os Métodos não farmacológicos de alívio da dor são preconizados pela Organização Mundial de Saúde (OMS) em seu Manual de "Boas práticas de Atenção ao Parto e ao Nascimento" e classificados como "condutas que são claramente úteis e que deveriam ser encorajadas" (Organização Mundial de Saúde, 1996).

Com base na Organização Mundial de Saúde (OMS) e Visando promover assistência humanizada no ciclo gravídicopuerperal, o Ministério da Saúde instituiu o Programa de Humanização no Pré-Natal e Nascimento (PHPN) pela Portaria/GM n. 569 do Ministério da Saúde, em $1^{\circ}$ de junho do ano de 2000 no Brasil. O programa busca promover o adequado acompanhamento do parto e puerpério, e os serviços de saúde devem organizar-se para garantir o cuidado com dignidade a mulher e ao recém-nascido (Brasil, 2014).

Considerando que o uso dos métodos não farmacológicos de alívio da dor no trabalho de parto traz uma abordagem humanizada no atendimento e ajuda as mulheres a vivenciarem esse momento de uma forma mais confortável e menos traumática, justifica-se a relevância de se estudar a percepção das mulheres com o uso desses métodos. Nessa perspectiva, o presente estudo teve como questionamento norteador: qual a percepção das puérperas sobre o uso de métodos não farmacológicos para alívio da dor no trabalho de parto? Para tanto, possui como objetivo: descrever a percepção das puérperas acerca do uso dos métodos não farmacológicos para alívio da dor durante o trabalho de parto e parto. 


\section{Metodologia}

Trata-se de uma pesquisa de campo, estudo descritivo com abordagem qualitativa. Os estudos com métodos qualitativos requerem a interpretação por parte dos pesquisadores por meio de suas opiniões e reflexões acerca do fenômeno em estudo (Pereira et al, 2018).

O estudo foi conduzido em uma maternidade pública do município de Recife - PE. Essa instituição foi escolhida por ser considerada referência no atendimento as gestantes classificadas para baixo risco obstétrico. Participaram do estudo 10 puérperas que fizeram uso de métodos não farmacológicos para alívio da dor durante o trabalho de parto.

Os critérios de inclusão foram: ser maior de 18 anos de idade, ter idade gestacional igual ou superior a 37 semanas. E como critério de exclusão: parturientes que evoluíram com óbito fetal ou recém-nascido com malformação grave e submetidas à cesariana de emergência. O número de participantes foi estabelecido por meio da saturação de dados, que foi atingido na décima entrevista, à medida que conseguiu responder ao objeto de estudo e as respostas começaram a se repetir.

A coleta de dados ocorreu nos meses de Outubro a Novembro de 2018. Para a abordagem das puérperas optou-se por ir à maternidade em dias alternados, para uma coleta de dados com puérperas assistidas em plantões diferentes.

A entrevista foi realizada em uma sala reservada no próprio setor de alojamento conjunto com a presença da participante e da pesquisadora. Utilizou-se um roteiro semiestruturado, cujas respostas foram gravadas e posteriormente transcritas na íntegra, para assim serem analisadas. A entrevista foi embasada na seguinte questão: Qual a sua experiência com a utilização dos métodos não farmacológicos de alívio da dor? Pergunta que foi aprofundada de acordo com a necessidade e características do estudo.

A análise dos depoimentos realizou-se por meio da técnica de Análise do Conteúdo de Bardin, que é definida como uma técnica de análise das comunicações que utiliza procedimentos sistemáticos e objetivos de descrição do conteúdo das mensagens e apresenta três etapas propostas: pré-análise, exploração do material e tratamento dos resultados obtidos e interpretação (Bardin, 2011).

A pesquisa foi realizada após aprovação pelo Comitê de Ética e Pesquisa do Instituto de Medicina Integral Professor Fernando Figueira- IMIP - CAAE N 68012817.0.0000.5201.

As participantes foram esclarecidas sobre o estudo: objetivo da pesquisa, importância, riscos e benefícios. Foi garantido o direito de recusa, desistência a qualquer momento do estudo, não havendo prejuízo ou interferência na sua terapêutica. Foi garantido o anonimato e o sigilo das informações, assim os nomes dos participantes da pesquisa são fictícios. As participantes assinaram o Termo de Consentimento Livre e Esclarecido (TCLE), em duas vias, sendo uma via para as mesmas e outra via para a pesquisadora. A pesquisa respeitou a Resolução do Conselho Nacional de Saúde - nº 466/2012.

\section{Resultados}

A seguir são apresentadas as categorias que emergiram a partir da leitura e observação dos dados. Os tópicos aqui expostos representam as experiências das puérperas que utilizaram pelo menos algum tipo de método não farmacológico de alívio da dor no parto. Surgiram quatro ideias centrais que foram categorizadas segundo Bardin: 1- conhecimento sobre os métodos não farmacológicos de alívio da dor. 2- sensação de bem estar e alívio da dor. 3- diminuição do tempo de trabalho de parto. 4- valorização do acompanhante.

\section{Categoria 1 - Conhecimento sobre os métodos não farmacológicos de alívio da dor}

Os depoimentos revelaram que as puérperas conheciam e receberam algum tipo de orientação sobre os métodos não farmacológicos para alívio da dor, estas aconteceram no período gestacional com o acompanhamento pré-natal, expressas por experiências próprias, bem como reveladas por familiares e amigos. 
"Conheci os métodos por amigas, na internet, e nas palestras do pré-natal pela Enfermeira" (GIRASSOL).

"Eu conhecia, vi na internet as melhores possibilidades para ter filho normal e também no pré-natal minha médica falou sobre eles" (JASMIN).

"Eu conhecia do meu primeiro filho e no pré-natal teve palestra com a Enfermeira também " (LÍRIO).

\section{Categoria 2 - Sensação de bem estar e alívio da dor}

A dor do trabalho de parto pode ser percebida de diferentes formas, variando entre cada mulher. Um dado recorrente nas falas foi o alivio das dores do trabalho de parto, como se percebe nos relatos a seguir.

"Ajudou muito nas dores, principalmente quando você faz o agachamento, porque quando tá deitada a dor vem mais forte e fazendo exercícios você consegue suportar melhor" (LÍRIO).

““" Eu achei interessante, diferente, pra não ficar só na cama e ainda ajudou muito no meu parto. Só fiquei na cama pra ter ele. Diminuiu minha dor, foi mais rápido, e menos doloroso. "Foi muito bom, teve uma parte que aliviava e até consegui cochilar e me acalmar" (VIOLETA).

"Muito bom a respiração e o banho morno me ajudou a relaxar bastante. Alivia muito a dor, ficar parada ou deitada é bem pior" (ORQUÍDEA).

"Ajuda você a sair do desespero, porque você fica na ansiedade pra ter logo e o exercício ajuda você a se distrair uт роисо" (ROSA).

\section{Categoria 3 - Diminuição do tempo de trabalho de parto}

A utilização dos métodos não farmacológicos, de acordo com os depoimentos das puérperas, atuou acelerando o trabalho de parto, desta forma, contribuindo com a diminuição do tempo de trabalho de parto.

"O cavalinho e o banho me ajudou bastante, porque acelerou mais as contrações e dilatou mais rápido" (JASMIN).

"Foi uma experiência única, eu acho que acelerou o trabalho de parto, foi mais rápido, principalmente quando eu agachei" (VIOLETA).

"Achei muito bom, porque ajudou ele a vir mais rápido" (LÍRIO).

“A evolução fica mais rápida, eu cheguei com $2 \mathrm{~cm}$ aí, andei, andei, andei, já foi logo pra 7cm” (ROSA).

\section{Categoria 4 - Valorização do acompanhante}

Ter sido acompanhada por alguém fez com que as puérperas tivessem uma experiência positiva, ressaltando a importância do acompanhante durante todo o período de trabalho de parto e parto.

"É muito bom que agora o acompanhante pode ficar com agente, nos meus outros filhos eu já fiquei sozinha" (ROSA).

"Minha acompanhante me ajudou a fazer massagem nas minhas costas, a agachar e a pessoa não fica só" (JASMIN).

"Com o acompanhante é melhor, alguém que você já conhece e não fica lá sozinha” (LÍRIO). 


\section{Discussão}

As orientações ministradas a gestante e/ou à parturiente são os recursos que mais colaboram para maior autonomia durante o trabalho de parto (Alves et al., 2015). Durante o pré-natal um espaço de educação em saúde deve ser criado, a fim de possibilitar o preparo da mulher para viver a gestação e o parto de forma positiva, integradora, enriquecedora e feliz. O grupo de gestantes é um referencial importantíssimo no contexto educativo e promotor da saúde da mulher. Este reforça a promoção da saúde mediante a discussão sobre temas variados, possibilitando à mulher conhecer o seu corpo e aumentar a segurança e tranquilidade durante a gestação e o parto (Cabral et al., 2013).

O acompanhamento durante o pré-natal deve ser a primeira medida visando o processo de parturição humanizado. Neste aspecto, a enfermeira que atua sob a perspectiva do parto humanizado desenvolve um papel relevante no acompanhamento das condições maternas e fetais, no processo de orientação da gestante quanto a sua participação ativa no trabalho de parto e parto e no preparo quando de sua passagem pelo centro obstétrico (Reis et al., 2016). Na assistência à parturiente, o enfermeiro deve instituir condutas embasadas cientificamente que valorizem o processo fisiológico do nascimento e parto (Feijão et al., 2017).

O movimento de humanização da assistência ao parto a nível mundial vem influenciando a busca por medidas não farmacológicas, pois estas promovem o alívio da dor, garantem segurança a parturiente na vivência do trabalho de parto e consequentemente, um parto mais saudável (Sartori et al., 2011).

De acordo com a Diretriz Nacional de Assistência ao Parto Normal estratégias e métodos não farmacológicos de alívio da dor no trabalho de parto devem ser implementados e sempre que possível ser oferecido às mulheres, proporcionando assim, um novo modelo de assistência ao parto (Brasil, 2017).

O contexto em que a parturiente se insere para o processo de trabalho de parto é o elemento que mais condiciona a sua dor. Sendo assim, a assistência prestada durante esse processo deve colaborar para que a mulher tenha um bom enfrentamento, tornando o momento menos doloroso, visto que a falta de atenção da equipe de saúde, a exposição de sua intimidade, a falta de alguém que lhe traga segurança (acompanhante) e o excessivo uso de métodos invasivos podem trazer grandes traumas à mulher e constituir uma violência consentida que só aumenta a percepção dolorosa e faz a mulher rejeitar uma nova experiência de parto normal (Alves et al., 2015).

A oferta dos métodos não farmacológicos é considerados alternativas para o alívio da dor, que surgem a partir das necessidades das parturientes em adotar métodos saudáveis em meio a uma vivência de pura sensibilidade que é o parto, diante da vontade da mulher e da redefinição de uma assistência obstétrica digna, evitando o uso de ferramentas institucionais que interferem diretamente na experiência do evento do parto (Almeida et al., 2015).

A Diretriz Nacional de Assistência ao Parto Normal traz recomendações em relação às estratégias e métodos não farmacológicos de alívio da dor no trabalho de parto, sendo elas: Sempre que possível deve ser oferecido à mulher a imersão em água para alívio da dor; os gestores nacionais e locais devem proporcionar condições para o redesenho das unidades de assistência ao parto visando a oferta da imersão em água para as mulheres no trabalho de parto; técnicas de massagem que tenham sido ensinadas aos seus acompanhantes devem ser apoiadas e realizadas, bem como técnicas de relaxamento (Brasil, 2017).

O processo de gestar e parir desperta muita ansiedade e estresse para a saúde do binômio mãe-filho, principalmente pelo medo do parto, e a auriculoterapia se mostra eficaz na redução da ansiedade na fase ativa do trabalho de parto (Manfetoniet al, 2018)

Um ensaio clínico randomizado envolvendo 178 participantes com idade gestacional de 30 - 32 semanas e uma gravidez normal. As participantes foram submetidas a 20 minutos de exercícios na bola suíça. O estudo concluiu que houve uma redução da sensação dolorosa nas participantes. Além disso, o estudo utilizou regressão logística que mostrou diminuição 
da dor em relação a alguns fatores, como na posição vertical parto, cesárea ou método de parto vaginal, bem como administração de ocitocina (Shirazi et al., 2019).

Os benefícios provenientes do uso da bola suíça no trabalho de parto são inúmeros e relaciona-se com o fato da posição vertical permitir que a força da gravidade associada ao alinhamento do eixo fetal com a bacia materna, favoreça a descida e a progressão do feto no canal de parto. Dessa forma, os efeitos da posição e movimentação materna durante o trabalho de parto podem relacionar com a diminuição da dor na região lombar, melhora da circulação materno-fetal, aumenta a intensidade das contrações uterinas, diminuem a duração do trabalho de parto, auxiliando assim na descida e insinuação do feto, como também diminuir as taxas de trauma perineal e episiotomia (Gois et al., 2020).

O banho de chuveiro, a movimentação durante o trabalho de parto e a massagem são consideradas métodos terapêuticos simples, de baixo custo e de fácil aplicabilidade, que ajudam a parturiente na diminuição da tensão, do medo e da sensação dolorosa, proporcionando conforto e favorecer a formação de vínculo com a equipe de enfermagem (Cortes et al., 2015).

Uma revisão sistemática realizada por Lawrence et al. incluiu 25 ensaios clínicos randomizados e controlados com o total de 5.218 mulheres. Os resultados encontrados foram redução da duração do trabalho de parto em torno de uma hora para as parturientes que deambularam ou adotaram posições verticais, não sendo observados efeitos negativos para a mãe e o recém-nascido. Os autores concluíram que as parturientes devem ser incentivadas a deambularem e adotarem posições mais confortáveis na primeira fase do trabalho de parto (Lawrence et al., 2013).

A Lei Federal n 11.108, de 07 de abril de 2005 conhecida como a Lei do Acompanhante, determina que os serviços de saúde do SUS, da rede própria ou conveniada, são obrigados a permitir à gestante o direito à presença de acompanhante durante todo o período de trabalho de parto, parto e pós-parto. A Lei determina ainda que esse acompanhante seja indicado pela mulher (Brasil, 2005). A presença do acompanhante no trabalho de parto ajuda a mulher positivamente, inclusive com redução dos níveis de dor. A presença do companheiro ou de alguém de confiança traz a sensação de conforto e cuidado, que coopera para maior segurança da mulher. Quando a participação ativa do acompanhante acontece, ele passa da posição de uma simples presença, para assumir o papel de provedor do suporte a parturiente (Pereira et al., 2016).

Entre as atividades de suporte realizadas por ele estão as medidas de conforto físico e de apoio emocional como: massagens, deambulação, oferta de líquidos/alimentos, incentivo a posições verticalizadas e utilização de exercícios respiratórios (Souza \& Gualda, 2016). A presença do acompanhante facilita a comunicação entre a parturiente e o profissional que lhe presta cuidados, trazendo-lhe satisfação e segurança na tomada de decisão (Dias et al., 2018).

A revisão sistemática conduzida por Hodnett et al. incluiu 22 ensaios clínicos randomizados e controlados com 15.288 mulheres, demonstrou que o suporte contínuo intraparto contribui para maior probabilidade de parto vaginal espontâneo, melhores experiência de parto, melhores índice de apgar no quinto minutos, redução do tempo de trabalho de parto, menor probabilidade de analgesia, cesariana, parto vaginal instrumental. Os autores concluíram que o apoio contínuo traz benefícios significativos para as mulheres e seus bebês, todas as mulheres devem receber suporte contínuo durante todo o trabalho de parto e o parto (Hodnet et al., 2013).

\section{Considerações Finais}

Analisar a percepção do uso de métodos não farmacológicos para alívio da dor no parto para a mulher permitiu visualizar a experiência pessoal de cada mulher, identificando os efeitos positivos dos métodos de alívio durante o processo de parturição. As mulheres relatam que o uso dos métodos não farmacológicos foi importante uma vez que acelerou o trabalho de parto, diminuindo a dor e valorizou também o suporte contínuo, ressaltando o papel do acompanhante no trabalho de parto e parto. 
Diante disso, a busca de conhecimento sobre a percepção das puérperas que usaram os métodos não farmacológicos no alívio da dor no parto foi importante para refletirmos sobre o cuidado prestado as mulheres. Dessa maneira, evidencia-se a importância de uma atenção integral e individualizada, contemplando os aspectos emocionais, culturais, sociais e psicológicos da parturiente e de sua família.

Por ser uma um campo da saúde que está em crescimento, destaca-se a importância dos Profissionais enfermeiros buscarem qualificação na área, a fim de prestar uma assistência diferenciada e de qualidade, uma vez que eles são os profissionais que possuem um grande acesso a mulher desde o período pré-natal até o momento do parto, sendo assim, profissionais determinantes na construção do conhecimento, bem como no empoderamento da mulher e de sua família.

Nesse sentido, faz-se necessário o desenvolvimento de novas pesquisas e estudos científicos com alto grau de evidências, em especial estudos clínicos, capazes de modificar e subsidiar a atuação e implementação dos métodos não farmacológicos na prática assistencial.

\section{Referências}

Almeida, J. M., Acosta, L. G., \& Pinhal, M. G. (2015). The knowledge of puerperae about non-pharmacological methods for pain relief during childbirth. REME: Revista Mineira de Enfermagem, 19(3), 711-717.

Alves, C. C., Cavalcante, M. N. B., Sampaio, A. C. C., Aragão, H. L., Oliveira, E. M., \& Teixeira, M. A. (2015). Humanização do parto a partir de Métodos não farmacológicos para alívio da dor: Relato de experiência. SANARE Suplemento. 2447-5815, V.14.

Bardin, L. (2011). Análise de conteúdo: Edições 70.

Brasil. (2017). Diretrizes Nacionais de Assistência ao Parto: Relatório de recomendação. Ministério da Saúde. Comissão Nacional de Incorporação de Tecnologias no SUS. Brasília.

Brasil. (2005). Lei $n^{\circ} 11.108$ de 07 de abril de 2005. Altera a Lei no 8.080, de 19 de setembro de 1990. Garante às parturientes o direito à presença de acompanhante durante o trabalho de parto, parto e pós-parto imediato, no âmbito do Sistema Único de Saúde - SUS. Diário Oficial da União.

Cabral, F. B., Hirt, L. M., \& Sand, I. C. P. V. (2013). Atendimento pré-natal na ótica de puérperas: da medicalização à fragmentação do cuidado. Revista . Esc. Enferm. USP, 47(2):281-7.

Côrtes, C. T., Santos, R. C. S., Caroci, A. S., Oliveira, S. G., Oliveira, S. M. J. V., \& Riesco, M. L. G. (2015). Implementation methodology of practices based on scientific evidence for assistance in natural delivery: a pilot study. Revista Esc. Enferm USP, 49(5):716-724.

Dias, E. G. et al. (2018). Eficiência de métodos não farmacológicos para alívio da dor no trabalho de parto normal. Enfermagem em Foco, ISSN 2357-707X.

Feijão, L. B. V., Boeckmann, L. M. M., \& Melo, M. C. (2017). Conhecimento de Enfermeiras Residentes Acerca das Boas Práticas na Atenção ao Parto. Enfermagem em Foco, 8(3): 35-39.

Gois, F. da L. M., Vieira, J. B. B., Freitas, L. A. L., Andrade, S. M. de, Cunha, M. A., Verde, R. M. C. L., \& Oliveira, E. H. de. (2020). The use of the Swiss ball to relieve pain in labor: a systematic review. Research, Society and Development, 9(7), e50973783.

Hodnett, E. D., Gates, S., Hofmeyr, G. J., \& Sakala, C. (2013). Continuous support for women during childbirth. Cochrane Database Syst Rev. (2):CD003766.

Lawrence, A., Lewis, L., Hofmeyr, G. J., \& Styles, C. (2013). Maternal positions and mobility during first stage labour. Cochrane Database Syst Rev.

Mafetoni, R. R., Rodrigues, M. H., Jacob, L. M. D. S., \& Shimo, A. K. K. (2018). Effectiveness of auriculo therapy on anxiety during labor: a randomized clinical trial. Revista latino - americana de enfermagem.

Ministério da Saúde. Parto, aborto e puerpério: assistência humanizada à mulher. Secretaria de Políticas de Saúde. Área Técnica de Saúde da Mulher. Brasília.

Ministério da Saúde. Cadernos HumanizaSUS: Humanização do parto e do nascimento. Brasília.

Moraes, M. S. T. (2010). Aplicabilidade de estratégias não farmacológicas para alívio da dor em parturientes: revisão integrativa. J. Nurse UFPE on line, 4(3 Suppl 1):S1070-5.

Organização Mundial de Saúde. (1996). Maternidade segura: assistência ao parto normal: um guia prático: relatório de um grupo técnico. Genebra. Pereira, A. $\quad$ S. $\quad$ et al. (2018). Metodologia da pesquisa científica. https://repositorio.ufsm.br/bitstream/handle/1/15824/Lic_C\%20omputacao_Metodologia-Pesquisa-Cientifica.pdf?sequence=1

Pereira, S. S., Oliveira, I. C. M. S., Santos, J. B. S., \& Carvalho, M. C. M. P. (2016). Parto natural: a atuação do enfermeiro diante da assistência humanizada. Actas de Saúde Coletiva, 10(3):199-213. 
Research, Society and Development, v. 10, n. 5, e30210514659, 2021

(CC BY 4.0) | ISSN 2525-3409 | DOI: http://dx.doi.org/10.33448/rsd-v10i5.14659

Reis, C. S. C., Souza, D. O. M., Nogueira, M. F. H., Progianti, J. M., \& Vargens, O. M. C. (2016). Análise de partos acompanhados por enfermeiras obstétricas na perspectiva da humanização do parto e nascimento. J. Res. Fundam. Care, 8(4):4972-4979.

Sartori, A.L et al. (2011). Estratégias não farmacológicas de alívio à dor durante o trabalho de parto. Enferm. Glob.10(21). ISSN 1695-6141.

Sescato, A. C., Souza, S. R. R. K., \& Wall, M. L. (2008). Os cuidados não-farmacológicos para alívio da dor no trabalho de parto: orientações da equipe de enfermagem. Cogitare Enfermagem, 13(4):585-90.

Shirazi, M. G., Kohan, S., Firoozehchian, F., \& Ebrahimi, E. (2019). Experience of Childbirth With Birth Ball: A Randomized Controlled Trial. International Journal of Women's Health and Reproduction Sciences; 7(3):301-305

Souza, S. R. R. K., \& Gualda, D. M. R. (2016). A experiência da mulher e de seu acompanhante no parto em uma maternidade pública. Rev. Texto Contexto Enferm. 25(1):1-9 